\title{
REVIEW
}

\section{Breeding strategies for increasing yield potential in super hybrid rice}

\author{
Shihua CHENG (ه), Xiaodeng ZHAN, Liyong CAO \\ China National Rice Research Institute, Hangzhou 310006, China
}

\begin{abstract}
Super hybrid rice breeding is a new breeding method combining semi-dwarf breeding and heterosis breeding using germplasm and gene-environment interactions. This paper reviews the breeding strategies of super hybrid rice breeding in China, focusing on the utilization of heterosis of indica and japonica subspecies, construction of ideal plant architecture and pyramiding of disease resistant genes in restorer lines. To develop super hybrid rice, considerable effort should be made to explore genes related with high yield, good quality, resistance to pests and diseases, tolerance to stresses. Molecular breeding methods in combination with crossing techniques should be adopted in super hybrid rice breeding.
\end{abstract}

Keywords super hybrid rice, breeding strategies, yield potential

\section{Importance of rice improvement in China}

Rice is a staple food in China and accounts for about $27 \%$ of total crop area planted. It feeds more than $60 \%$ of the population, contributing nearly $40 \%$ of total calorie intake $^{[1]}$ and is essential for ensuring food security in China.

China has successful experience in the using germplasm and genes to increase the yield potential in rice. In the last century, the development of semi-dwarf cultivars in the 1950s and application of heterosis in the 1970s were two revolutionary breakthroughs in rice breeding. There were two key genes in these breakthroughs, namely semi-dwarf gene $s d_{1}$, cytoplasmic male sterility (CMS) gene from wild rice and its restoring gene $R f$. With advancement of rice breeding, and high demand for food from the increasing population in China, a national collaborative research program on super hybrid rice breeding was established by

Received September 15, 2015; accepted October 23, 2015

Correspondence: shcheng@mail.hz.zj.cn the Ministry of Agriculture, P. R. China in 1996. Super hybrid rice is a new type of rice combining an ideal plant type with strong heterosis. The breeding of super hybrid rice in China had three key periods, 1996-2000, 20012005 and 2006-2015, and the yield targets of single season cropping of rice were defined as $10.5,12$ and $13.5 \mathrm{t} \cdot \mathrm{hm}^{-2}$ for each period, respectively.

Since 1996, 146 super rice cultivars, including 92 super hybrids, have been released provincially or nationally and planted on a large scale. Super hybrid rice has contributed significantly to rice production, and to rice yield per unit area in China, reaching historical levels (Fig. 1). The breeding technique using heterosis combined with ideal plant type has become one of the unique technologies in super hybrid rice breeding in the world ${ }^{[2]}$.

The definition of a super hybrid rice is a hybrid cultivar with ideal plant architecture, high yield potential, high grain quality and disease resistance. Super hybrid rice cultivars need three outstanding features: plant morphology with straight and upright leaves, moderate tillering, medium plant height and big panicles; physiological function with high photosynthetic efficiency, strong root vigor and compatible source and sink; genetic potential for a combination of high yield, good quality, stress tolerance and disease resistance traits. The combination of subspecies heterosis with the ideal plant architecture is the key feature, and two-line and three-line hybrids are the two major methods used in super hybrid rice breeding ${ }^{[3-5]}$.

In this paper, we review and provide some new insights into the breeding strategies, especially in utilization of germplasm and genes in super hybrid rice breeding programs, and evaluate the prospects for super rice breeding in the future.

\section{Breeding strategies for super hybrid rice}

\subsection{Utilization of indica and japonica subspecies heterosis}

Heterosis is a general phenomenon whereby the first generation produced by crossing two genetically different 


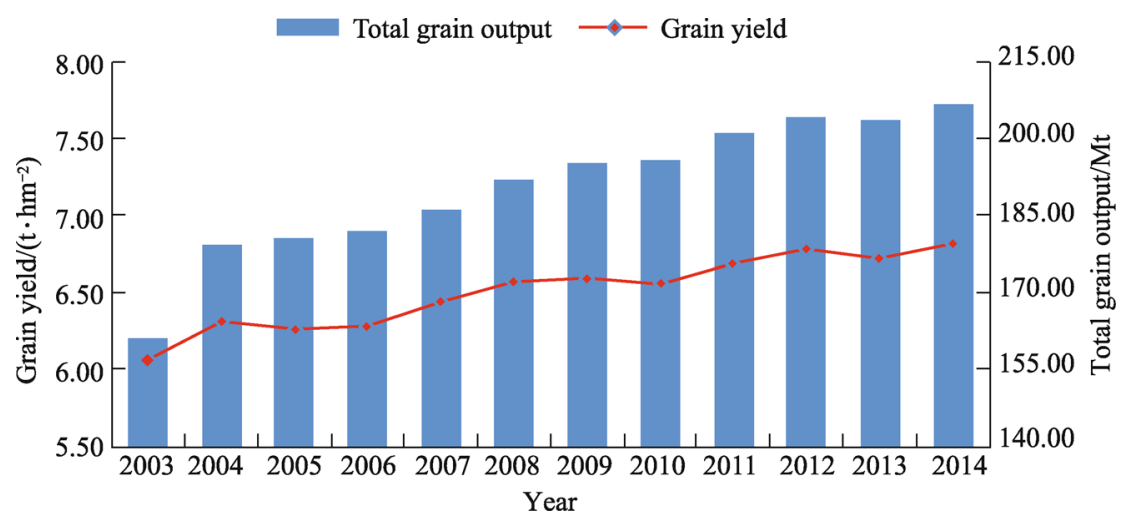

Fig. 1 Rice yield and production in China (2003-2014)

parents has superior growth potential, viability, fertility, resistance, yield and quality than either of the two parents. Rice heterosis was first proposed by Jones in 1926, but the studies on indica and japonica rice hybrids were not carried out until the 1950s. In the studies of inter-subspecies heterosis, the key questions are how to rapidly improve the setting rate of the $F_{1}$ generation of inter-subspecific hybrids, to combine the advantages of indica and japonica subspecies and to apply inter-subspecies heterosis in rice production. Yang et al. ${ }^{[6]}$ carried out a study on the utilization of indica and japonica inter-subspecies heterosis. Their results indicated that the setting rate of the $F_{1}$ generation between indica and japonica rice can be increased to normal levels through artificial selection. Recently, the genetic mechanism of $S_{5}$, a wide compatibility gene, was completely described at the molecular level ${ }^{[7]}$, which revealed the molecular mechanism of fertility regulation in indica/japonica hybrids, and provided a new application potential for the inter-subspecies hybrid breeding.

Although the question of how indica and japonica rice subspecies separated from wild rice is unresolved, considerable evidence from morphology, cytology and genome analysis has highlighted the divergence the indica and japonica rice subspecies. Theoretically, there are two ways to use indica and japonica inter-subspecies heterosis, namely direct and indirect utilization. Direct utilization is to use the wide compatibility genes to overcome sterility between indica and japonica rice, and to fully activate the strong heterosis between the subspecies. The indirect way is partially using inter-subspecies heterosis through different means. In breeding practice, indica and japonica belong to two rice subspecies, their $F_{1}$ generations often have stronger nutritional advantage, longer growth period and higher plant height than the parents, and often possess a reproductive barrier such as partial pollen abortion and low seed setting rate, so that inter-subspecies heterosis is difficult to exploit in breeding practice.

Indica and japonica subspecies heterosis has been adopted gradually in rice breeding in China since the 1970s. A large number of inbred rice cultivars have been bred by multiple crosses of indica and japonica combined with continuous generational selection. Since the 1980s, breeders have paid more attention to breeding restorer lines from the progeny of indica/japonica hybrids. However, both the breeding of inbred rice cultivars and restorer lines was largely based on random crossing and a large number of selections. Also, the best introgression ratio for indica/ japonica heterosis needs to be established. Our research indicated that the heterosis of the $\mathrm{F}_{1}$ generation in typical indica/indica and japonica/japonica crosses were weak because of low gene exchange between subspecies. However, excessive gene exchange between indica and japonica was harmful in harmonization of vegetative and reproductive growth, and not conducive to increased yield. In our previous research, we found that the selection of intermediate type parents is important in breeding super hybrid rice, especially for indica/intermediate and intermediate/intermediate crosses, because these two methods can coordinate the yield heterosis and ideal plant architecture to the maximum degree ${ }^{[8,9]}$. Many intermediate restorer lines were developed through the method of introgression crossing between indica and japonica, and have been used in our super hybrid rice breeding program (Table 1).

\subsection{Construction of ideal plant architecture}

Plant architecture refers to a group of morphological characteristics related to the plant spatial arrangement and yield potential. Donald first proposed the concept of the ideal plant architecture as a biological model which can express expected features under particular environmental conditions in $1968^{[10]}$. As a typical breeding practice for rice genetic improvements of plant architecture or plant type, Yang et al. ${ }^{[11]}$ suggested that ideal plant architecture is the ideal combination of plant morphological and functional traits for improvement of photosynthetic efficiency at an individual plant level. At the population level, ideal plant architecture means the ideal arrangement 
Table 1 Four super hybrids developed using intermediate restorer lines derived from introgression crosses of indica and japonica

\begin{tabular}{lcc}
\hline Super hybrid & Combination & Pedigree of the restorer lines \\
\hline Xieyou 9308 & Xieqingzao A/ R9308 & R9308: C57 (j)//No. 300 $(j) /$ IR26( $i)$ \\
Guodao 1 & Zhong 9A/R8006 & R8006: IRBB60//T2070/Duoxi 1 \\
Guodao 3 & Zhong 8A/R8006 & T2070: WL1312(j)/Lunhui 422 $(m) / /$ Minghui 63 $(i)$ \\
Guodao 6 & Neixiang 2A/R8006 & - \\
\hline
\end{tabular}

Note: $i, j$ and $m$ represent indica, japonica and intermediate type of rice, respectively.

of yield-related photosynthesis elements in space to achieve the highest level of biological and economical outputs. Specifically, breeding for the ideal plant type can create new plant architecture by regulating geometry and coordinated spatial arrangement between different individuals, so they can adapt to the natural and artificial environments better under population conditions, and population structure and light situation can also be improved. The minimum extinction co-efficient extends the canopy photosynthesis time as well as improving the dry matter production capacity as much as possible. Based on the ecological conditions in different regions, some ideal plant type models of super rice including super inbred and super hybrid for different regions have been proposed (Table 2).

Using the generalized definition of plant architecture, the morphology and function of roots is also an important plant architecture related trait. As a consequence of the complex growth environment and limitation in research methods for root systems, genetic studies on root traits have lagged behind other traits in rice. Studies on plant architecture and its breeding have often been restricted to the aboveground parts, such as stems and leaves ${ }^{[12,13]}$. Recently, with the advances in super rice breeding, the incompatibly between big panicles and plant presenility is becoming increasingly obvious. Therefore, the importance of the rice root system and its inclusion in breeding priorities should be given greater attention.

Studies on root-related genes have made useful progress in recent years. The root morphological traits include length, number of thick roots, dry weight, density of length, volume, penetration ability, depth and the ratio of root to canopy, among others. Using a recombinant inbred line population of Xieyou 9308 and two backcross populations derived from that population, Liang et al. ${ }^{[14]}$ detected a total of eight QTLs related to root traits at the heading stage. Among them, a pleiotropic QTL related to several root traits was repeatedly detected between RM180 and RM5436 on the short arm of chromosome 7 (Fig. 2). On further research, a QTL qRL7 was fine mapped between the markers InDel11 and InDel17 $(657.35 \mathrm{~kb}$ interval) on chromosome 7 , which played a crucial role in root length ${ }^{[15]}$.

Mapping root traits showed the complexity of the genetic system of roots. Mutants are ideal for gene identification and cloning, but suitable mutants are difficult to create, especially for roots. These are not only difficult to identify, but also many root lethal mutants are difficult to obtain and preserve. As a consequence of this limitation there are few cloned genes related to root traits.

Table 2 Ideal plant type models of super rice proposed by different institutions in China

\begin{tabular}{lcc}
\hline Unit & Ideal plant type & Representative cultivar \\
\hline Shenyang Agricultural University & Erect and large panicle & Shennong 265 (inbred) \\
Guangdong Academy of Agricultural Sciences & Earlier growing with deep root system & Shengtai 1 (inbred) \\
Sichuan Agricultural University & Weighted panicle & Ilyou 162 (hybrid) \\
Hunan Hybrid Rice Research Center & Erect and long canopy leaves & Liangyou E32 (hybrid) \\
China National Rice Research Institute & Vigor at late growth stage & Xieyou 9308 (hybrid) \\
\hline
\end{tabular}

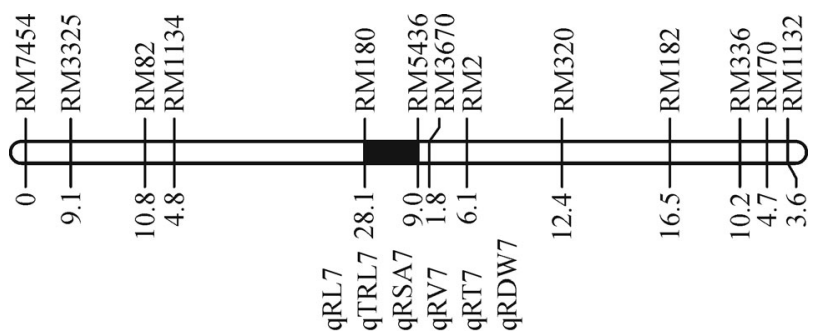

Fig. 2 Composite interval mapping of QTL for the seven root traits on chromosome 7 
Rice root breeding is defined as developing new rice cultivars through genetic improvement of root systems according to different perspectives and different demands and objectives. Based on the different breeding aims, rice root breeding can be divided into the following aspects: high yield, abiotic stress tolerance and grain quality. However, the current studies on root genes are insufficient to provide any assistance in rice root breeding. Therefore, the approach has been the conscious improvement of root traits which can influence grain yield and rice yield directly or indirectly, with the basic idea to create root characteristics with ideal architecture, lasting and stable function and wide adaptability, coordinated with the optimization of physiological and ecological traits of aboveground parts, and to achieve the objectives of high yield, stable yield, high efficiency, good quality and ecological suitability.

As with breeding for plant architecture of aboveground parts, rice root breeding also has two categories or aspects: root morphology and physiological breeding. Recent studies have shown for the root system of rice that bigger is not better, rather an appropriate structure and function is important. The function of the root system needs to be durable and efficient, but not excessively large and presenility. The breeding aim for super hybrid rice is to properly control the number of roots, with the most effective method being to maintain the number of nonrooting sections appropriately. Some research on the rice root system has also shown that increasing the length and branching of roots is helpful in increasing the surface area of the root system ${ }^{[16]}$. Although we had found that super hybrid rice cultivars Xieyou 9308 and Guodao 6 were better than their parents with regard to root depth, distribution and vigor ${ }^{[17,18]}$, these aspects are still preliminary and there needs to be further systematic research to develop a key index, identification methods and clarify the relationship between gene regulation and growth of aboveground parts.

\subsection{Pyramiding of disease resistance genes in restorer lines}

Apart from the introgression cross of indica and japonica, we also studied the disease resistance by molecular identification and pyramiding different resistant genes into restorer lines. Rice blast and bacterial blight are two major diseases of rice. If the rice is infected by the blight bacteria, its leaves will become necrotic and leaf photosynthetic function will significantly decrease. Rice panicleneck blast can prevent the transportation of photosynthates to the panicle and lead to rice panicle death. In previous research, we identified a major blast resistant gene $P i-25$ on chromosome 6 from the native cultivar Gumei 2, which has resistance to both leaf and panicle blast disease. Furthermore, random amplified polymorphic DNA markers located in the flaking region of Pi-25 were converted into two STS markers SK17 and SA7, which have been used in breeding of blast resistant restorer lines. In addition, molecular marker pTA248 was used in the rapid identification of bacteria blight resistance gene $\mathrm{Xa} 21$. Using molecular assisted selection method, two restorer lines, Zhonghui 8006 and Zhonghui 111, were created (Table 3). Through marker identification, we found the disease resistance genotype of Zhonghui 8006 to be $\mathrm{Xa4}+$ $x a 5+x a 13+X a 21$ and Zhonghui 111 to be Xa21 + xa5 + $P i-25$. Among them, rice blast resistance gene $P i-25$ was derived from Gumei 2 and bacterial blight resistance genes $X a 4, x a 5, x a 13$ and Xa21 were derived from IRBB54 and IRBB60, which was obtained from the International Rice Research Institute. Besides pyramiding the blast and bacterial blight resistance genes, these two restorer lines and super hybrid Guodao 6 also show ideal plant types (Fig. 3, Fig. 4).

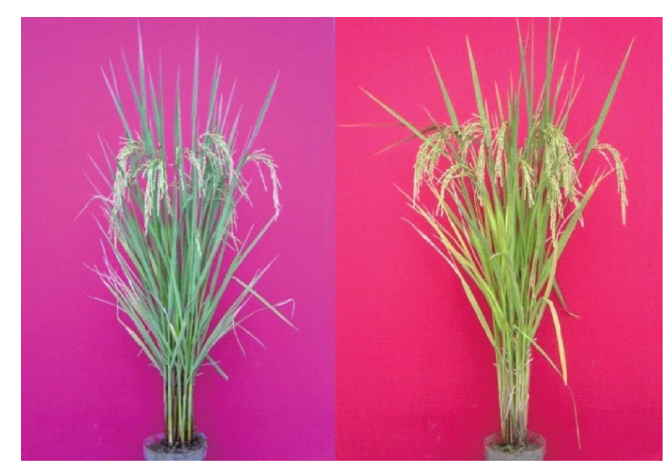

Fig. 3 Ideal plant type of two key restorer lines used in super hybrid rice breeding

Table 3 Origins of two key restorer lines used in super hybrid rice program

\begin{tabular}{lcc}
\hline Name of restorer lines & Zhonghui 8006 & Zhonghui 111 \\
\hline Plant variety right No. & CNA20030473.9 & CNA20050210.7 \\
Pedigree & IRBB60/L1 & IRBB54/R32 \\
& L1: T2070/Duoxi 1 & R32: 300(j)//G3-11/Minghui 63 \\
& T2070: WL1312(j)/Lunhui 422(m)//Minghui 63 $(i)$ & Gumei 2/zhong 156 \\
Index of japonica & 0.25 & $x a 5+X a 21+P i-25$ \\
Genotype of disease resistance & $X a 4+x a 5+x a 13+X a 21$ & 2 \\
Released cultivars & 5 & 0.39 \\
\hline
\end{tabular}




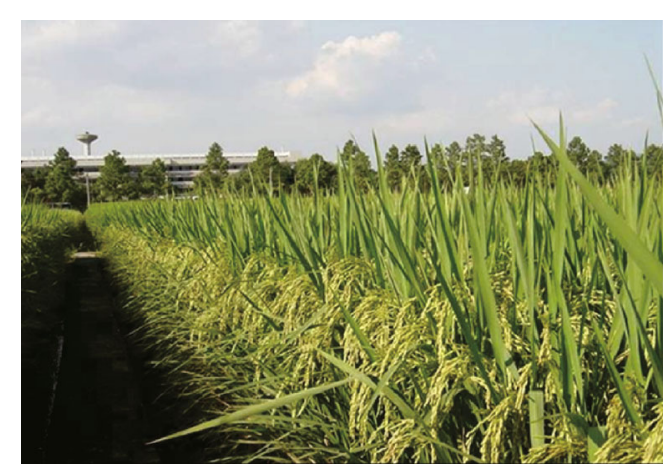

Fig. 4 Guodao 6, a super hybrid rice planted on a large scale in China

\section{Three prospects}

The core of super hybrid rice breeding is the effective use of germplasm and multi-genes recombination. The first stage of super hybrid rice breeding involved exploring and utilizing germplasm is to increase yield potential. With the exception of the cultivars which had disease resistance genes introduced by marker assisted selection, there are no successful examples in the direct utilization of genes related yield and plant architecture in super hybrid rice. This indicates that favorable genes and linkage markers useful in super hybrid rice breeding directly are currently insufficient and progress in genetic studies has not been sufficient to support super hybrid rice molecular breeding.

In addition to increased yield, the target of super hybrid rice breeding is to provide better quality and adaptability. The basic breeding strategy of new super hybrid rice is to incorporate rice germplasm resources, functional genomics and molecular breeding technology into the breeding program, strengthening the study of the biological basis of important traits and gene exploration to develop new rice hybrids with high resistance to pests, tolerance to abiotic stress, nutrition efficiency, high yield and good quality. Indeed the comprehensive improvement of the traits of rice is needed to make the label, "super", become true. If we want to achieve new breakthroughs in hybrid rice breeding, molecular techniques must be enhanced. Currently in rice, marker assisted selection has only been successful for disease resistance breeding, so exploration and utilization of rice genes related to good quality, high yield, disease resistance and development of their linkage markers should be further strengthened. It is necessary to improve the efficiency of resource and gene utilization and strengthen super hybrid rice breeding with high insect resistance, wide ecological adaption and mechanized production through the combination of molecular and crossing techniques.

Acknowledgements This work was supported by the Chinese Super Rice Breeding Program (201403002), Ministry of Agriculture of the People's
Republic of China, and the National Natural Science Foundation of China (31101209)

Compliance with ethics guidelines Shihua Cheng, Xiaodeng Zhan, and Liyong Cao declare that they have no conflict of interest or financial conflicts to disclose.

This article is a review and does not contain any studies with human or animal subjects performed by any of the authors.

\section{References}

1. Cao L Y, Zhan X D, Chen S G, Feng Y, Wu W, Shen X, Cheng S. Breeding methodology and practice of super rice in China. Rice Science, 2010, 17(2): 87-93

2. Cheng S H, Cao L Y, Zhuang J Y, Chen S G, Zhan X D, Fan Y Y, Zhu D F, Min S K. Super hybrid rice breeding in China: Achievements and prospects. Journal of Integrative Plant Biology, 2007, 49(6): 805-810 (in Chinese)

3. Yang S R, Zhang L B, Chen W F, , Xu Z J, Wang J M. Theories and methods of rice breeding for maximum yield. Journal of Shenyang Agricultural University, 1996, 27(1): 1-7 (in Chinese)

4. Yuan L P, Tang C D. Retrospections, present status and prospects in hybrid rice breeding. China Rice, 1999, (1): 3-6 (in Chinese)

5. Cheng S H, Zhai H Q. Comparison of some plant type components in super high-yielding hybrids of inter-subspecies rice. Acta Agronomica Sinica, 2000, 26(6): 713-718 (in Chinese)

6. Yang S R, Shen X Y, Gu W L, Cao D J. Study on indica/japonica hybrid rice breeding (No. 2). Acta Agronomica Sinica, 1962, 1(2): 97-102 (in Chinese)

7. Yang J, Zhao X, Cheng K, Du H, Ouyang Y, Chen J, Qiu S, Huang J, Jiang Y, Jiang L, Ding J, Wang J, Xu C, Li X, Zhang Q. A killerprotector system regulates both hybrid sterility and segregation distortion in rice. Science, 2012, 337(6100): 1336-1340

8. Cheng S H, Mao C Z, Zhan X D. Construction of double haploid (DH) and recombinant inbred line (RIL) population of indicajaponica hybrid and their differential in indica and japonica property. Chinese Journal of Rice Science, 2001, 15(4): 257-260 (in Chinese)

9. Cheng S H. Innovation and development of rice breeding for super high yield in China. Journal of Shenyang Agricultural University, 2007, 38(5): 647-651 (in Chinese)

10. Xue D W, Qian Q. Genetic basis and resources innovation of super rice breeding in China. Journal of Shenyang Agricultural University, 2007, 38(5): 667-675

11. Yang S R. Zhang L B, Wang J M. The theory and method of ideal plant morphology in rice breeding. Scientia Agricultura Sinica, 1984, 3: 6-12(in Chinese)

12. Zhu D F, Lin X Q, Cao W X. Comparison of leaf photosynthetic characteristics among rice hybrids with different leaf rolling Index. Acta Agronomica Sinica, 2001, 27(3): 329-333 (in Chinese)

13. Zhu D F, Lin X Q, Cao W X. Effects of deep roots on growth and yield in two rice varieties. Scientia Agricultura Sinica, 2001, 34(4): 429-432 (in Chinese)

14. Liang Y S, Zhan X D, Wang H M, Gao Z Q, Lin Z, Chen D B, Shen X H, Cao L Y, Cheng S H. Locating QTLs controlling several adult 
root traits in an elite Chinese hybrid rice. Gene, 2013, 526(2): 331335

15. Wang H M, Xu X M, Zhan X D, Zhai R, Wu W, Shen X, Dai G, Cao $\mathrm{L}$, Cheng S. Identification of $q R L 7$, a major quantitative trait locus associated with rice root length in hydroponic conditions. Breeding Science, 2013, 63(3): 267-274

16. Wu W M, Cheng S H. Significance and prospects of breeding for root system in rice (Oryza sativa L.). Chinese Journal of Rice
Science, 2005, 19(2): 174-180 (in Chinese)

17. Wang X, Tao L X, Yu M Y, Huang X L. Physiological model of super hybrid rice Xieyou 9308. Chinese Journal of Rice Science, 2002, 16(1): 38-44 (in Chinese)

18. Cheng S H, Cao L Y, Chen S G, Zhu D F, Wang X, Min S K, Zhai H Q. Conception of late-stage vigor super hybrid rice and its biological significance. Chinese Journal of Rice Science, 2005, 19(3): 280-284 (in Chinese) 